\title{
Energy Transfer Study of the Cylindrical Interface Formed by Asymmetric Isoprene-Methyl Methacrylate Diblock Copolymers Bearing a Dye at the Junction
}

\author{
Jian Yang, Xudong Lou, John G. Spiro and Mitchell A. Winnik ${ }^{*}$ \\ Department of Chemistry, University of Toronto, 80 St. George Street, \\ Toronto, Ontario, Canada M5S 3H6
}

\section{Supporting Information}

\section{DETERMINATIN OF INTERFACE THICKNESS FOR DYE-LABLED ASSYMMETRIC PI-PMMAS - CONSIDERATION OF DOUBLE DONOR LIFETIMES}

In this supporting information, we present a data analysis of experimental donor fluorescence decays by the MC method under the assumption that the donor-labeled PI-PMMA can be characterized by two discrete lifetimes (1.7 ns and $43.4 \mathrm{~ns})$. We assumed there is no energy transfer between the short-lifetime component (1.7 ns) and the labeled acceptors. Under this assumption, the equations for energy transfer (eqs 4, 5 and 6) can be modified as

or

$$
\begin{aligned}
& I(t)=\frac{A_{1}}{N_{D}^{0}} \exp \left(-\frac{t}{\tau_{D 1}}\right) \sum_{j=1}^{N_{D}^{0}} \exp \left(-\frac{3}{2} \frac{t}{\tau_{D 1}}\langle|\kappa|\rangle^{2} \sum_{k=1}^{N_{A}^{0}}\left(\frac{R_{0}}{r_{j k}}\right)^{6}\right)+A_{2} \exp \left(-\frac{t}{\tau_{D 2}}\right) \\
& I(t)=\frac{A_{1}}{N_{D}^{0}} \exp \left(-\frac{t}{\tau_{D 1}}\right) \sum_{j=1}^{N_{D}^{0}} \exp \left(-\frac{3}{2} \frac{t}{\tau_{D 1}} \sum_{k=1}^{N_{A}^{0}} \kappa_{j k}^{2}\left(\frac{R_{0}}{r_{j k}}\right)^{6}\right)+A_{2} \exp \left(-\frac{t}{\tau_{D 2}}\right)
\end{aligned}
$$

where $\tau_{\mathrm{D} 1}(43.4 \mathrm{~ns})$ and $\tau_{\mathrm{D} 2}(1.7 \mathrm{~ns})$ are the two donor lifetimes. $A_{1}$ and $A_{2}$, are the two coefficients that reflect the fractions of the two decay lifetime components. All other variables have the same physical meanings as those in eqs 4, 5 and 6. We use eq s1 (or eq s2, depending on how we consider the dipole orientation) to fit the experimental decays in same way as we used eqs 4 and 6 as described in the text of the paper. In the data analysis described here, the two coefficients, $A_{1}$ and $A_{2}$, were allowed to float. The optimized value of interface thickness at each acceptor concentration was retrieved from the minimum of the $\chi^{2}$ curve against the probing values of interface thickness. 


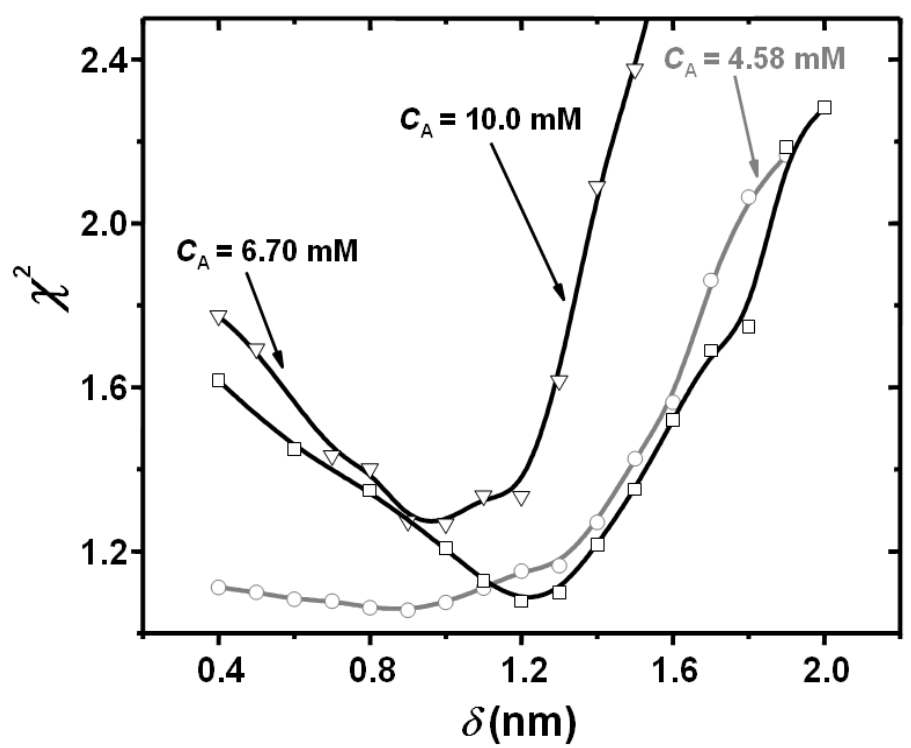

Figure s1 $\chi^{2}$ surface plot for samples (No. 2, 3, and 4) listed in Table 2. The preaveraged orientation factor $\langle|\kappa|\rangle^{2}=0.476$ was used in FRET calculations (eq s1). The acceptor concentration for each curve is indicated in the figure. Two donor lifetimes are used in the experimental decay fittings.

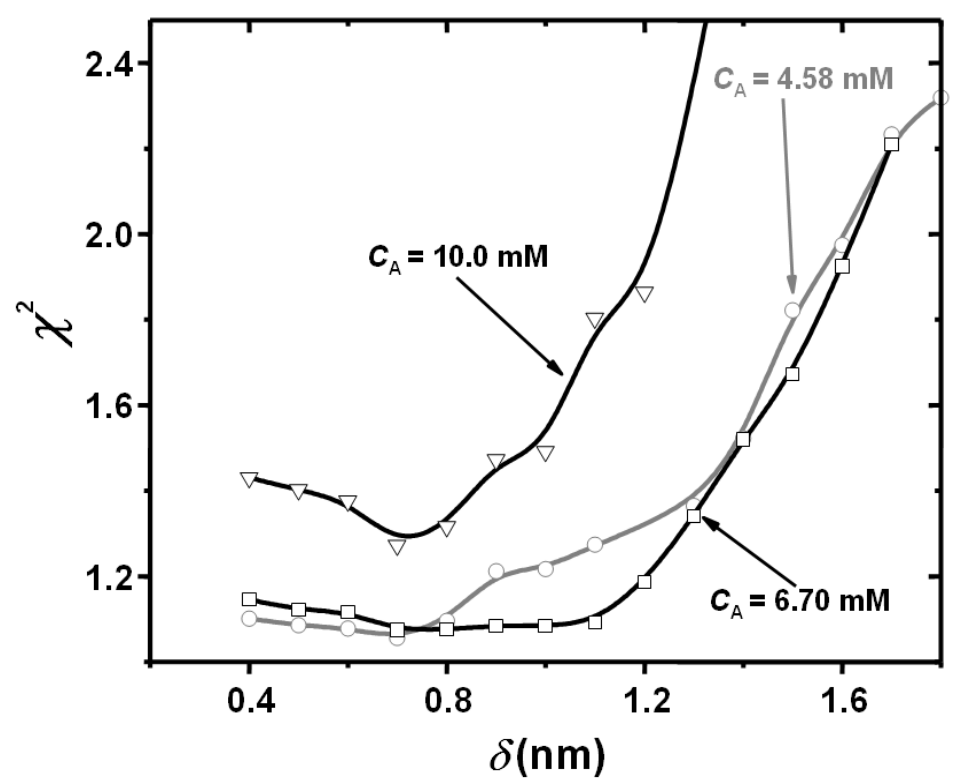

Figure s2 $\chi^{2}$ surface plot for samples (No. 2, 3, and 4) listed in Table 2. Individual random dipole orientations were considered in the FRET calculations (eq s2). The acceptor concentration for each curve is indicated in the figure. Two donor lifetimes are used in the experimental decay fittings. 
Figures s1 shows $\chi^{2}$ surface plots for the fits under the assumption of a pre-averaged orientation factor $\left(\langle|\kappa|\rangle^{2}=0.476\right)$. For each acceptor concentration, we found similar $\chi^{2}$ curves compared with Figure 5 in the paper. The similar $\chi^{2}$ curves indicate that the existence of the second short lifetime (1.7 ns) has only a negligible effect on the retrieved value of the interface thickness.

Figure s2 shows $\chi^{2}$ surface plots for the fits under the assumption of individual random dipole orientations. The $\chi^{2}$ curves are also similar to the corresponding curves shown in Figure 7. Based on these results, we conclude that the neglecting the second lifetime (1.7 ns) in the analyses of fluorescence decay curves brought little change to the retrieved interface thickness for our synthetic dye-labeled PI-PMMA samples. 